\title{
ON WEAKLY ORDERED SYSTEMS
}

\section{RICHARDSON}

1. Introduction. By a weakly ordered system we mean a system $D$ of elements $x, y, \cdots$ with a binary relation $>$ such that

$$
x>y \text { implies } x \neq y
$$

and

$$
x>y \text { implies } y>x \text { is false. }
$$

The statement " $x>y$ " may be read " $x$ dominates $y$." Transitivity is not assumed; a transitive weakly ordered system is a partially ordered system. By a solution of a weakly ordered system is meant a set $V$ of elements of $D$ such that (a) $x \in V$ and $y \in V$ implies $x<y$ is false and (b) $x \in D-V$ implies $y>x$ for some $y \in V$. The concept of solution was introduced in J. von Neumann and O. Morgenstern, Theory of games and economic behavior, Princeton, 1944, where it is proved that a weakly ordered system which is strictly acyclic ${ }^{1}$ possesses a solution which is unique, and for which a construction is given. This result suggests the problem of finding conditions for the existence and uniqueness of solutions of weakly ordered systems in general. The simplest examples show that if cycles exist neither the existence nor the uniqueness of solutions can be expected in all cases. For example, the system of three elements $a>b>c>a$ has no solution, while the system of four elements $a>b>c>d>a$ has the two solutions $(a, c)$ and $(b, d)$. The purpose of this note is to prove the existence of solutions for certain non-acyclic systems. The proof will itself provide a method of construction for the solutions. Zermelo's axiom of choice, the well-ordering theorem, and transfinite induction will be used. The result presented below is a contribution to the general problem suggested above rather than to the theory of games. For the hypothesis of the theorem below precludes transitivity completely; that is, it precludes the existence of three elements $a, b, c$, such that $a>b, b>c$, and $a>c$. This restriction is too severe for the theory of games, just as is the assumption of transitivity. The problem remains open for weakly ordered systems which are not strictly acyclic but also do not satisfy the hypothesis of the theorem below.

Presented to the Society, September 17, 1945; received by the editors August 10, 1945.

1 See loc. cit. pp. 590-600 for definitions and proof. 
2. The theorem. We shall find it convenient to represent the weakly ordered system $D$ by an oriented 1-complex or linear graph whose vertices are in 1-1 correspondence with the elements of $D$ and such that two vertices $x$ and $y$ are joined by an oriented 1-cell $x y$, oriented from $x$ toward $y$ if and only if the element corresponding to $x$ dominates the element corresponding to $y$. This oriented graph will also be denoted by $D$, as well as its set of vertices, and we shall denote indiscriminately by $x, y, \ldots$ either the elements of $D$ or the vertices of the graph with which they are identified. The graph $D$ of a weakly ordered system is not the most general oriented graph; it contains no loops consisting of an oriented 1-cell whose initial and terminal vertices are the same (condition (1) above), and no two vertices are joined by more than one 1-cell (condition (2) above). A graph is called even if all its unoriented ${ }^{2} 1$-cycles contain an even number of 1-cells (or vertices). We shall prove the following theorem.

THEOREM. If the graph $D$ is even, then solutions exist.

Of course, the hypothesis of this theorem implies that all cycles, in the sense of von Neumann and Morgenstern, loc. cit., are even, but not conversely.

3. A lemma on graphs. We shall use the following known lemma whose proof we include for the sake of completeness and to facilitate the construction of examples by the reader.

Lemma (KöNIG). ${ }^{3}$ The set of vertices of an even graph $D$ can be decomposed into two disjoint sets $P$ and $Q$ such that every 1-cell of $D$ joins $a$ vertex of $P$ to one of $Q$.

Proof. Select a definite vertex $x$ of any (connected) component of $D$. Put a vertex $y$ of the same component into $P$ or $Q$ according as the unoriented 1-chains joining $x$ and $y$ have an odd or even number of 1 -cells. If a vertex $y$ could be joined to $x$ by two 1-chains, one with an odd and the other with an even number of 1-cells, then there would exist an unoriented 1-cycle with an odd number of 1-cells, contrary to hypothesis. This may be done for each component of $D$. This proves the lemma.

4. Proof of the theorem for finite systems. For simplicity, we give the proof first for finite systems.

2 That is, modulo 2.

3 D. König, Über Graphen und ihre Anwendung auf Determinantentheorie und Mengenlehre, Math. Ann. vol. 77 (1916) pp. 453-465. Cf. also D. König, Theorie der endlichen und unendlichen Graphen, Leipzig, 1936. 
Let $P$ and $Q$ be two classes selected according to the lemma. Let the elements $p$ of $P$ be ordered by means of a subscript index ranging over a lower segment of (finite) ordinal numbers. Let $P_{0}=0$ and $Q_{0}=Q$. We now define sets $N_{k}, P_{k+1}, R_{k}, Q_{k+1}$ inductively for $k=0,1,2,3, \cdots$. Let $N_{k}$ be the set of all elements of $P-P_{k}$ which are not dominated by any element of $Q_{k}$. If $N_{k}=0$, let $V=P_{k} \cup Q_{k}$. If $N_{k} \neq 0$, let $p_{\alpha_{k}}$ be the first element of $N_{k}$, that is, the element with the lowest ordinal subscript. Let $P_{k+1}=P_{k} \cup\left[p_{\alpha_{k}}\right]$. Let $R_{k}$ be the set of all elements of $Q_{k}$ which are dominated by $p_{\alpha_{k}}$. Let $Q_{k+1}=Q_{k}-R_{k}$.

Since the set $P$ is finite, it is clear that there is a least ordinal $\lambda$ for which $N_{\lambda}=0$. We shall prove that

$$
V=P_{\lambda} \cup Q_{\lambda}
$$

is a solution.

Notice the obvious relations

$$
\begin{aligned}
& P_{0} \subseteq P_{1} \subseteq P_{2} \subseteq \cdots \subseteq P_{\lambda} \subseteq P, \\
& Q=Q_{0} \supseteq Q_{1} \supseteq Q_{2} \supseteq \cdots \supseteq Q_{\lambda},
\end{aligned}
$$

(6) $Q=\left(\cup_{\beta<\lambda} R_{\beta}\right) \cup Q_{\lambda}$ where the sets $\left(\cup_{\beta<\lambda} R_{\beta}\right)$ and $Q_{\lambda}$ are disjoint.

We must prove: (a) if $x \in V, y \in V$, then $x<y$ is impossible; (b) if $x \in D-V$, then there exists an element $y \in V$ such that $x<y$.

Proof of (a). Suppose $x<y$.

(i) Suppose $x$ and $y$ belonged both to $P_{\lambda}$ or both to $Q_{\lambda}$. Since $P_{\lambda} \subseteq P$ and $Q_{\lambda} \subseteq Q$, this would contradict the property of $P$ and $Q$ given in the lemma of König.

(ii) If $x \in P_{\lambda}$ and $y \in Q_{\lambda}$, then $x=p_{\alpha \gamma} \in N_{\gamma}$ for some $\gamma<\lambda$ and hence is not dominated by any element of $Q_{\gamma}$, and, a fortiori, of $Q_{\lambda}$. This is a contradiction.

(iii) If $x \in Q_{\lambda}$ and $y \in P_{\lambda}$, then $y=p_{\alpha \gamma}$ for some $\gamma<\lambda$. Then $x \in R_{\gamma}$ and cannot be an element of $Q_{\gamma+1}=Q_{\gamma}-R_{\gamma}$, nor, a fortiori, of $Q_{\lambda}$. This is a contradiction.

PROOF OF (b). Suppose no such $y$ existed.

(iv) If $x \in P \cap(D-V)$, then $x \notin P-P_{\lambda}$ since $N_{\lambda}=0$. Hence $x \in P_{\lambda}$. Therefore $x \in V$. This is a contradiction.

(v) If $x \in Q \cap(D-V)$, then, by (6), $x \in R_{\gamma}$ for some $\gamma<\lambda$. Then there exists a $y$ such that $x<y \in P_{\gamma+1} \subseteq P_{\lambda} \subseteq V$. This is a contradiction.

This completes the proof.

5. Proof of the theorem for infinite systems. We indicate the modifications necessary in the infinite case.

We assume that all finite unoriented cycles have an even number of 1-cells. By the component to which a vertex belongs we mean the 
set of all vertices which can be joined to it by finite unoriented 1chains. Then the lemma of König holds as before. Now, let the elements $p$ of $P$ be well-ordered by means of a subscript index ranging over a lower segment of ordinal numbers. The construction of the sets $N_{k}, P_{k+1}, R_{k}, Q_{k+1}$ will be as before for finite $k$. But now it may happen that $N_{k}=0$ for no finite $k$.

Consider any ordinal number $\gamma$. We shall construct the sets $N_{\gamma}, P_{\gamma}$, $Q_{\gamma}, R_{\gamma}$, and so on, by transfinite induction.

Case I. If $\gamma$ is a limit ordinal and $N_{\beta}=0$ for no $\beta<\gamma$ then let $P_{\gamma}=U_{\beta<\gamma} P_{\beta}$ and $Q_{\gamma}=\bigcap_{\beta<\gamma} Q_{\beta}$. Let $N_{\gamma}$ be the set of those elements of $P-P_{\gamma}$ not dominated by any element of $Q_{\gamma}$. Let $p_{\alpha \gamma}$ be the first element of $N_{\gamma}$, and let $P_{\gamma+1}=P_{\gamma} \cup\left[p_{\alpha_{\gamma}}\right]$. Let $R_{\gamma}$ be the set of those elements of $Q_{\gamma}$ dominated by $p_{\alpha \gamma}$, and let $Q_{\gamma+1}=Q_{\gamma}-R_{\gamma}$.

Case II. If $\gamma$ is not a limit ordinal, it has an immediate predecessor $\gamma-1$. If $N_{\beta}=0$ for no $\beta<\gamma$, let $p_{\alpha_{\gamma-1}}$ be the first element of $N_{\gamma-1}$. Let $P_{\gamma}=P_{\gamma-1} \cup\left[p_{\alpha_{\gamma-1}}\right]$. Let $R_{\gamma-1}$ be the set of those elements of $Q_{\gamma-1}$ dominated by $p_{\alpha_{\gamma-1}}$. Let $Q_{\gamma}=Q_{\gamma-1}-R_{\gamma-1}$. Let $N_{\gamma}$ be the set of those elements of $P-P_{\gamma}$ not dominated by elements of $Q_{\gamma}$.

Let $\pi$ be the ordinal number of the set $P$ in the chosen well-ordering. Note that $\beta<\gamma$ implies $\alpha_{\beta}<\alpha_{\gamma}$. There must exist a least ordinal $\lambda$ such that $N_{\lambda}=0, \lambda \leqq \pi$. Let $V=P_{\lambda} \cup Q_{\lambda}$. It is readily seen that (4), (5), and (6) hold whether $\lambda$ is a limit ordinal or not. The proofs of (a) and (b) can now be seen to proceed as in the infinite case.

Brooklyn College 